\title{
Escala Abreviada para la Evaluación de la Ansiedad por Separación en Adultos (ASA-10) en Población Española
}

\author{
Abbreviated Scale for the Assessment of Adult Separation Anxiety Questionnaire in \\ the Spanish Population
}

\author{
Antonio Ruiz-García ${ }^{1}$, Óliver Jiménez ${ }^{2}$, Davinia M. Resurrección ${ }^{3}$ y Javier Fenollar-Cortés ${ }^{4}$
}

\begin{abstract}
Resumen
El trastorno de ansiedad por separación se caracteriza por un miedo o ansiedad excesivos e inapropiados para el desarrollo en relación con la separación de las personas a las que el individuo está unido. El objetivo del presente estudio fue examinar: estructura factorial, consistencia interna y validez convergente de la versión abreviada del ASA-27 con 403 individuos (55.8\% mujeres) de entre 18-86 años $(M=37.1$ y $D T=13.9)$ de España. El ASA-10 correlacionó significativamente con las subescalas del Inventario de Ansiedad EstadoRasgo, del Cuestionario de Apego Adulto y del Cuestionario de Dependencia Emocional ( $r s=.11$ a .63 , $p s<.05)$. Presenta una estructura unifactorial y una consistencia interna adecuada $(\omega=.98)$. El ASA-10 mostró una sensibilidad del $97.9 \%$ para los casos con dificultades de ansiedad clínica. Los resultados muestran que el ASA-10 es una medida fiable y válida de la ansiedad por separación en adultos.
\end{abstract}

Palabras clave: ansiedad por separación en el adulto, cuestionario de ansiedad por separación en adultos, escala reducida, propiedades psicométricas

\begin{abstract}
Separation anxiety disorder is characterized by developmentally inappropriate levels of excessive fear or anxiety concerning separation from those to whom the individual is attached to. The objective of the present study was to examine factorial structure, internal consistency and convergent validity of the abbreviated version of the ASA-27. The sample comprised 403 Spanish people (55.8\% female) aged between 18 and 86 years $(M=37.1 ; S D=13.9)$. ASA-10 was significantly related to all the State-Trait Anxiety Inventory, Adult Attachment Questionnaire and Emotional Dependency Questionnaire subscales ( $r s=.11$ to .63, $p s<.05$ ). ASA-10 presented a single-factor structure and appropriate internal consistency $(\omega=.98)$. ASA-10 showed a sensitivity of $97.9 \%$ for cases with clinical anxiety difficulties. In conclusion, the results show that the ASA10 is a reliable and valid measure of separation anxiety in adults.
\end{abstract}

Keywords: adult separation anxiety, shortened version, psychometric properties

\footnotetext{
${ }^{1}$ Doctor en Psicología. Departamento de Psicología. Facultad de Ciencias de la Educación. Universidad de Córdoba. Av. San Alberto Magno, s/n, 14071 Córdoba, España. Tel.: +34957218847. Correo: aruiz1@uco.es

2 Doctorando en Psicología. Universidad de Málaga. Facultad de Psicología. Campus Teatinos, 29071 Málaga, España. Tel.: +34644477898. Correo: oliverjjimenez@uma.es

${ }^{3}$ Doctora en Psicología. Departamento de Psicología. Universidad Loyola Andalucía. Avenida de las Universidades s/n, 41704 Dos Hermanas, Sevilla, España. Tel.: +34 955641600- ext 2423. Correo: dmresurreccion@uloyola.es

${ }^{4}$ Doctor en Psicología. Departamento de Psicología. Universidad Loyola Andalucía. Avenida de las Universidades s/n, 41704 Dos Hermanas, Sevilla, España. Tel.: +34 955641600- ext 2466. Correo: jfenollar@uloyola.es
} 


\section{Introducción}

El trastorno de ansiedad por separación en el adulto (TASA) se caracteriza por la presencia de ansiedad excesiva ante el alejamiento del hogar o de las personas a quienes el sujeto está vinculado, que resulta excesiva o inapropiada para el nivel de desarrollo del individuo que lo padece, es persistente y causa un malestar o afectación clínicamente significativa en áreas importantes de funcionamiento (DSM-5; American Psychiatric Association, 2013). En esta nueva versión del DSM se modifica la conceptualización del trastorno, al considerar que puede tener su inicio en la edad adulta y, además, una manifestación clínica de naturaleza diferente al trastorno de ansiedad por separación en la etapa infantil (TAS). En adultos, la ansiedad anticipatoria elevada ante la separación simbólica o real del hogar o figuras de apego principales propia del TASA (Deveci, 2019; Manicavasagar \& Silove, 2020a), puede manifestarse, entre otras posibles causas, a partir de una mudanza del domicilio familiar o la finalización de una relación (Mabilia, Di Riso, Liso, \& Bobbio, 2019). La persona sufre una ansiedad elevada, expresada también como preocupación intensa, por la integridad física o seguridad de las figuras de apego, motivando conductas de seguridad y conductas orientadas a permanecer en contacto continuo con ellas (Dowsett et al., 2020; Gesi et al., 2015; Manicavasagar \& Silove, 2020a; Posavac \& Posavac, 2017; Pozzi et al., 2014). Lo que genera un desajuste psicológico general (Román, Hodges, Palacios, Moreno, \& Hillman, 2015). Por ejemplo, padecer sintomatología como dolor de estómago ante la idea de separarse de la figura de apego y sentir temor a que algo pueda ocurrirle a esta si se separa de ella (Fernández-Marcos, 2014) o tener problemas para cambiar de ciudad por trabajo, hacer viajes fuera de la provincia por ocio (sola o acompañada) por la separación de las figuras de apego o contexto cercano, emanciparse del hogar familiar, entre otros (Ruiz \& Rando, 2015).

Siguiendo a Pini, Abelli y Bruschi (2014), el TASA ha mostrado una modesta asociación con apego ansioso y se ha relacionado con el trastorno dependiente de personalidad. Además, se ha encontrado relación entre dependencia emocional y ansiedad por separación en adultos en población española (Ruiz-García et al., 2019). Por otro lado, en población portuguesa (Ruiz-García et al., 2021) esta asociación se encontró solo en mujeres. Asimismo, en ambos estudios se ha observado que existía relación entre ansiedad por separación en adultos y ansiedad rasgo (predisposición a la ansiedad).

Además, el TASA presenta una elevada comorbilidad con otros cuadros clínicos tales como el trastorno depresivo mayor, trastorno por estrés postraumático, trastornos de la personalidad, trastorno de pánico, trastorno de ansiedad generalizada, trastorno bipolar I y II, o el trastorno ciclotímico (Gesi et al., 2015; Hale, Berg, \& Malhotra, 2018; Manicavasagar \& Silove, 2020b; Pozzi et al., 2014).

Dado el impacto en el funcionamiento general que se asocia con el trastorno de ansiedad por separación en edad adulta, y la elevada comorbilidad que presenta con otros cuadros clínicos, resulta muy conveniente el diseño y desarrollo de medidas específicas que permitan detectar y evaluar el trastorno en edad adulta. La primera entrevista clínica desarrollada para evaluar el TASA fue la Adult Separation Anxiety Semistructured Interview (ASA-SI; Manicavasagar, Silove y Curtis, 1997). Esta entrevista se elaboró a partir de los criterios diagnósticos que se recogían en el DSM-IV del TAS en la infancia y adolescencia. La ASA-SI contiene 27 ítems que deben ser respondidos teniendo como referencia los últimos tres meses a partir del momento de la evaluación. Basada en esta entrevista, posteriormente se publicó la escala Adult Separation Anxiety Questionnaire (ASA-27; Manicavasagar, Silove, Wagner y Drobny, 2003), la cual contiene los mismos 27 ítems con cuatro opciones de respuesta tipo Likert. Esta escala ha sido adaptada a población turca (Dirioz, Alkin, Yemez, Onur, \& Eminagaoglu, 2011), en población española (Ruiz-García et al., 2019) y, recientemente adaptada y validada en población portuguesa (Ruiz-García et al., 2021), mostrando unas propiedades psicométricas adecuadas, similares a las de la escala original.

A pesar de que la escala ASA-27 no es particularmente extensa, una corriente amplia de la psicometría y la psicología clínica propone el desarrollo de escalas cada vez más breves, sin que ello suponga una merma de sus cualidades 
psicométricas (Schweizer \& DiStefano, 2016). En este sentido, la dificultad de aplicar un rango amplio de escalas debido al tiempo que requieren los instrumentos extensos disminuye la posibilidad de actuar de manera temprana para disminuir el riesgo, evitar la aparición o reducir el efecto de un trastorno del comportamiento (Greer \& Liu, 2016). No obstante, a pesar de que el desarrollo de versiones abreviadas de los instrumentos existentes puede optimizar el tiempo, coste, esfuerzo, así como facilitar una primera aproximación a un determinado objetivo clínico, el proceso de reducción de escalas no está exento de riesgos, por lo que debe realizarse de manera rigurosa a nivel metodológico (para una revisión, Kruyen, Emons, \& Sijtsma, 2013).

La escala ASA-27 tanto en su versión original como en la adaptación a población española superan la consistencia interna de .90 , apareciendo la posibilidad de redundancia y de poder llevar a cabo una reducción de la escala (Streiner, 2003; Tavakol \& Dennick, 2011). Por todo ello, el objetivo del presente estudio fue desarrollar una versión abreviada del ASA-27 que pudiera ser empleada en entornos clínicos o con fines de investigación, conservando las propiedades psicométricas adecuadas. Así como, obtener la validez concurrente del cuestionario, utilizando distintos instrumentos que evalúen constructos similares como la ansiedad estado-rasgo o el apego (Ruiz-García et al., 2019), y que sea potencialmente útil para la detección de perfiles con riesgo de presentar sintomatología compatible con un TASA.

\section{Método}

\section{Participantes}

Para este estudio de diseño y análisis psicométrico se obtuvo una muestra que estuvo formada por 403 personas de ambos sexos $(55.8 \%$ mujeres y $44.2 \%$ hombres) de entre 18 y 86 años $(M=37.1, D T=13.9)$. Respecto al estado civil, el $57.8 \%$ era soltero/a, el $32.0 \%$ casado/a, el $6 \%$ separado/a, el $1.2 \%$ viudo/a, y el resto $(2.7 \%)$ no indicó estado civil. De la muestra, el $53.8 \%$ estaba activo laboralmente, el $28.8 \%$ estaba en periodo de formación, y el $11.7 \%$ estaba desempleado en el momento del estudio. En cuanto al nivel de formación, el $61.8 \%$ tenía un título universitario de grado o posgrado, el $28.5 \%$ había cursado bachillerato o formación profesional, y el resto no había alcanzado el nivel de estudios anterior (9.6\% de la muestra).

\section{Instrumentos}

Inventario de ansiedad estado rasgo (STAI; Spielberg, Gorsuch, \& Lushene, 1970; adaptación española de Buela-Casal, Guillén-Riquelme, \& Seisdedos-Cubero, 2015). Escala de 20 ítems con cuatro opciones de respuesta tipo Likert $(0=$ nada, $1=$ algo, 2 =bastante, $3=$ =mucho), que mide la ansiedad como estado (estado emocional transitorio, de naturaleza subjetiva, caracterizado por una hiperactividad del sistema nervioso autónomo) y como rasgo (propensión ansiosa a percibir las situaciones como amenazadoras). La escala ha mostrado en esta muestra una buena fiabilidad tanto en la escala «ansiedad estado» $(\alpha=.93)$ como en la escala «ansiedad rasgo» $(\alpha=.87)$.

Cuestionario de apego adulto (CAA; Melero \& Cantero, 2008). Escala de 40 ítems con seis opciones de respuesta tipo Likert respecto al grado de desacuerdo ( $1=$ completamente en desacuerdo, $2=$ bastante en desacuerdo, $3=$ algo en desacuerdo, 4=algo de acuerdo, 5=bastante de acuerdo, 6=completamente de acuerdo), con cuatro subescalas — cuya confiabilidad en la muestra del presente estudio se indica entre paréntesis-: (i) escala 1, baja autoestima, necesidad de aprobación y miedo al rechazo $(\alpha=.77)$; (ii) escala 2 , resolución hostil de conflictos, rencor y posesividad $(\alpha=.79)$; (iii) escala 3, expresión de sentimientos y comodidad con las relaciones $(\alpha=.70)$; y (iv) escala 4 , autosuficiencia emocional e incomodidad con la intimidad $(\alpha=.71)$.

Cuestionario de dependencia emocional (CDE; Lemos-Hoyos \& Londoño-Arrendondo, 2006). Escala de 23 ítems tipo Likert con 6 opciones de respuesta ( $1=$ completamente falso en mí, $2=$ la mayor parte falso en mí, $3=$ ligeramente más verdadero que falso, 4=moderadamente verdadero en mí, $5=$ la mayor parte verdadero en mí, 6=me describe perfectamente), en seis factores: (i) ansiedad por separación $(\alpha=.87)$, (ii) expresión afectiva de la pareja $(\alpha=.86)$, (iii) modificación de planes $(\alpha=.79)$, (iv) miedo a la soledad $(\alpha=.85)$, (v) expresión límite $(\alpha=.62)$, y (vi) búsqueda de atención $(\alpha=.71)$. 
Cuestionario de ansiedad por separación en el adulto (ASA-27; validación española de RuizGarcía et al., 2019). Escala de 27 ítems tipo Likert con cuatro opciones de respuesta relativas con el grado de ocurrencia ( $0=$ ocurre con mucha frecuencia, 1=ocurre con bastante frecuencia, $2=$ ocurre ocasionalmente, $3=$ no ocurre nunca). Evalúa ansiedad relacionada con la separación del objeto de vinculación o de los iguales. La escala genera una única puntuación total, y la consistencia interna es elevada en esta muestra $(\alpha=.91)$.

\section{Procedimiento}

La batería de cuestionarios se aplicó mediante muestreo de bola de nieve en población general española, administrándose secuencialmente en el mismo orden para todos los sujetos por dos profesionales formados específicamente en la administración de las pruebas. Para el muestreo se identificaron sujetos potenciales en la población mediante muestreo por conveniencia contactando con diversos centros de tratamiento psicológico privados y educativos de adultos, pidiendo a esos sujetos que reclutaran a otros participantes, produciéndose un reclutamiento de remisión en cadena. El procedimiento seguido para la adaptación de la escala original-queda recogido de manera detallada en Ruiz-García et al. (2019). Concretamente, se realizó un proceso de traducción-retrotraducción del inglés al castellano llevado a cabo por tres investigadores bilingües. Todos los procedimientos se ajustaron a las normas éticas del comité de investigación institucional y a la Declaración de Helsinki de 1964 y sus enmiendas posteriores o normas éticas comparables. Se obtuvo el consentimiento informado de todos los participantes adultos incluidos en el estudio. El protocolo del estudio fue aprobado por el Comité de Ética de Málaga (España), código interno: 1360-N-20.

\section{Estrategia de análisis}

La estrategia de análisis siguió tres fases consecutivas. En la primera fase, y de acuerdo con el procedimiento desarrollado en el artículo original de la escala en inglés (Manicavasagar et al., 2003), se replicaron los análisis estructurales y de consistencia interna, respetando los criterios de inclusión establecidos por los autores. En una segunda fase, se llevaron a cabo los análisis orientados al proceso de pulido de ítems para una versión reducida de la escala original (Kemper, Trapp, Kathmann Samuel, \& Ziegler, 2019). El pulido de ítems se llevó a cabo mediante un análisis factorial de ejes principales. La selección del análisis factorial exploratorio de ejes principales resulta particularmente deseable cuando hay una severa violación del supuesto de normalidad de los datos (Fabrigar, Wegener, MacCallum, \& Strahan, 1999), aplicando rotación oblicua Promax sin forzar la extracción de factores. Para la decisión respecto al número de factores, se retuvieron aquellos factores con un valor propio superior a uno. Además, se generaron gráficos de sedimentación para el apoyo de la decisión final de factores. Los criterios de inclusión de los ítems en la solución final fueron: (i) carga factorial superior a .60; (ii) ausencia de cargas cruzadas en dos dimensiones del constructo; y (iii) elevada consistencia interna de la estructura resultante (es decir, $\alpha$ Cronbach superior a .80). Adicionalmente, se calculó el $\alpha$ Cronbach de la escala al omitir cada uno de los ítems, siendo un criterio de inclusión que la consistencia interna no fuera superior a la escala sin omisión de ítems. En una tercera fase, se llevaron a cabo análisis de correlación entre la puntuación total de ambos modelos del ASA (versión original y versión reducida), y con el resto de las escalas para la validez convergente. Para interpretar el grado de efecto se consideró una correlación de .1 a .29 , como baja; de .3 a .49 , como media; y de .5 en adelante, como amplia (Cohen, Cohen, West, \& Aiken, 2002). Estos análisis fueron realizados con JASP versión 0.11.1 (JASP team, 2019).

\section{Resultados}

\section{Comparativa de la estructura factorial $y$ consistencia interna entre la escala original y la escala adaptada a población española}

Se calcularon los estadísticos descriptivos de cada uno de los ítems en la versión completa de la escala (ver Tabla 1). En un primer análisis, se exploró la matriz de correlación entre los ítems a través del cálculo del estadístico rho de Spearman $(\rho)$, con el fin de descartar aquellos con valores superiores a .80 que pudieran sugerir redundancia 
Tabla 1. Estadísticos descriptivos del ASA-27

\begin{tabular}{|c|c|c|c|c|c|}
\hline Summary of the scale items & $M$ & $D T$ & $M d n$ & Skew & Kurt \\
\hline Ítem 1 (Seguridad con personas cercanas) & 1.50 & 1.09 & 2 & -.01 & -1.29 \\
\hline Ítem 2 (Lejanía de casa) & .29 & .62 & 0 & 2.38 & 5.63 \\
\hline Ítem 3 (Objeto de seguridad en el bolso) & .58 & .95 & 0 & 1.49 & .98 \\
\hline Ítem 4 (Estrés ante un viaje largo) & .67 & .87 & 0 & 1.22 & 69 \\
\hline Ítem 5 (Sueño y pesadillas por separación) & .73 & .87 & 1 & 1.07 & .39 \\
\hline Ítem 6 (Estrés ante viaje de persona cercana) & .66 & .86 & 0 & 1.18 & .54 \\
\hline Ítem 7. (Molestia por interrupción de rutina) & .91 & .89 & 1 & .78 & -.07 \\
\hline Ítem 8 (Preocupación por intensidad de relación) & .70 & .80 & 1 & .96 & .28 \\
\hline Ítem 9 (Dolores o náuseas anticipatorias) & .56 & .79 & 0 & 1.27 & .77 \\
\hline Ítem 10 (Habla excesiva para mantener la cercanía) & .43 & .72 & 0 & 1.64 & 2.02 \\
\hline Ítem 11 (Preocupación por otros cuando están ausentes) & .62 & .78 & 0 & 1.23 & 1.09 \\
\hline Ítem 12 (Dificultades de sueño en soledad) & .79 & .96 & 0 & .99 & -.11 \\
\hline Ítem 13 (Mejoría sueño cuando personas cerca) & .7 & 1.00 & 0 & 1.20 & .15 \\
\hline Ítem 14 (Ansiedad por separación o lejanía de personas) & .76 & .87 & 1 & .95 & .10 \\
\hline Ítem 15 (Pesadillas por lejanía del hogar) & .24 & .58 & 0 & 2.67 & 7.30 \\
\hline Ítem 16 (Preocupación por pensamiento catastrofistas) & 1.21 & .95 & 1 & .48 & -.64 \\
\hline Ítem 17 (Molestias por cambios de rutina si afecta a relaciones) & .77 & .81 & 1 & .91 & .35 \\
\hline Ítem 18 (Preocupación por abandono de personas cercanas) & .85 & .88 & 1 & .91 & .19 \\
\hline Ítem 19 (Mejoría del sueño si hay luces encendidas) & .26 & .62 & 0 & 2.71 & 7.25 \\
\hline Ítem 20 (Evitación de soledad en casa) & .33 & .70 & 0 & 2.27 & 4.67 \\
\hline Ítem 21 (Ansiedad o ataque de ansiedad por lejanía de personas) & .40 & .75 & 0 & 1.91 & 2.78 \\
\hline Ítem 22 (Ansiedad por no contacto telefónico) & .49 & .81 & 0 & 1.65 & 1.94 \\
\hline Ítem 23 (Temor por abandono de alguien cercano) & .73 & .82 & 1 & .94 & .22 \\
\hline Ítem 24 (Ocurrencia de ataques de pánico ante soledad) & .37 & .70 & 0 & 1.96 & 3.24 \\
\hline Ítem 25 (Preocupación excesiva por pensamientos de daño ajeno) & .56 & .74 & 0 & 1.24 & 1.14 \\
\hline Ítem 26 (Comentarios negativos por habla excesiva) & .66 & .92 & 0 & 1.30 & .71 \\
\hline Ítem 27 (Preocupación por problemas ajenos a causa propia) & .37 & 64 & 0 & 1.63 & 1.87 \\
\hline
\end{tabular}

Nota. ASA-27=Cuestionario de Ansiedad por Separación en el Adulto (27 ítems); M=Media; Mdn=Mediana; Skew=Asimetría;

Kurt=Curtosis. Puntuación de los ítems va de 0 (no me ha sucedido nunca) a 3 (me sucede muy a menudo)

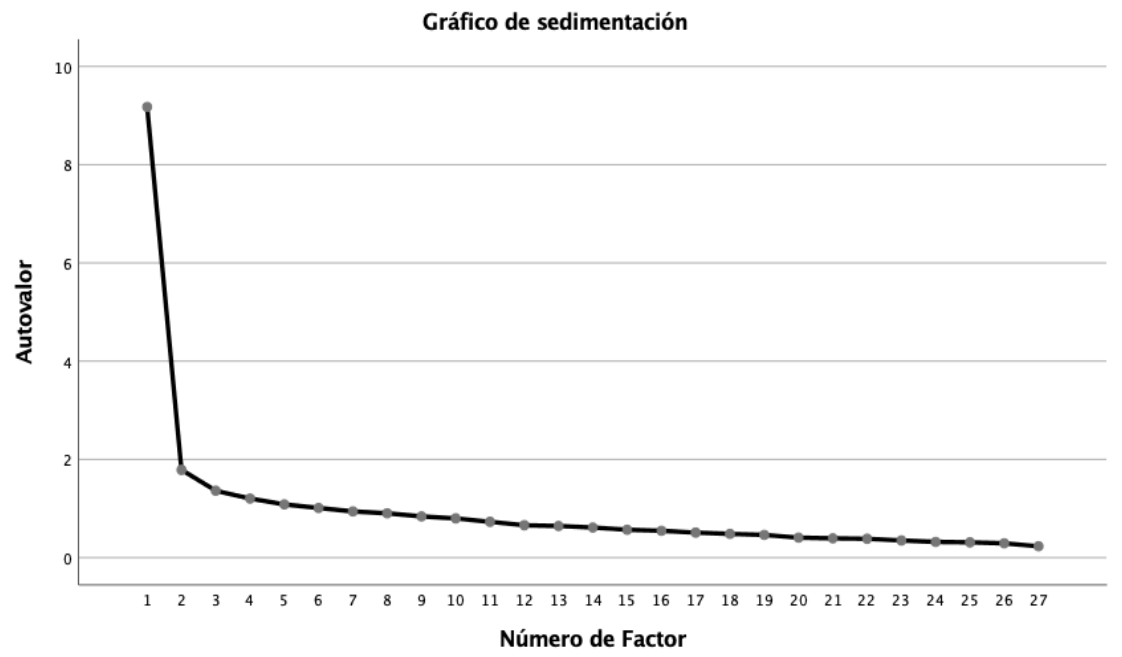

Figura 1. Gráfico de sedimentación del análisis factorial exploratorio por ejes principales de los 27 ítems de la escala ASA

(Madagascar et al. 2003). Los resultados mostraron correlaciones dentro de un rango que fue de $\rho=.05(p=.292)$ a $\rho=.65(p<.001)$, por lo que no se eliminó ningún ítem. Además, los resultados fueron similares a los del artículo original, con correlaciones entre .1 y .7, con la mayoría de los rangos entre .3 у .5 (en este estudio, los rangos que fueron del .25 a .50 supusieron el $70.4 \%$ de los resultados).
Las correlaciones entre los ítems y el resultado total de la escala se situaron dentro de un rango de $\rho=.34$ a $\rho=.67 \quad(p<.001)$, ligeramente inferior a los resultados en la versión original (de .42 a .84). Para los análisis de este trabajo, se ha calculado el cociente de Spearman dado que los valores no siguieron una distribución normal (KolmogorovSmirnov <.001, en todos los casos), mientras que en la versión original no se indicó el tipo de análisis. 
Tabla 2. Cargas factoriales por ítem tras extracción de factores por ejes principales en la escala ASA-27 sobre población española

\begin{tabular}{|c|c|c|}
\hline Ítems resumidos de la escala & Factor 1 & Factor 2 \\
\hline Ítem 1 (Seguridad con personas cercanas) & .357 & .117 \\
\hline Ítem 2 (Lejanía de casa) & .490 & .008 \\
\hline Ítem 3 (Objeto de seguridad en el bolso) & .486 & -.030 \\
\hline Ítem 4 (Estrés ante un viaje largo) & .613 & -.094 \\
\hline Ítem 5 (Sueño y pesadillas por separación) & .645 & -.030 \\
\hline Ítem 6 (Estrés ante viaje de persona cercana) & .684 & -.091 \\
\hline Ítem 7. (Molestia por interrupción de rutina) & .481 & -.442 \\
\hline Ítem 8 (Preocupación por intensidad de relación) & .501 & -.302 \\
\hline Ítem 9 (Dolores o náuseas anticipatorias) & .525 & -.114 \\
\hline Ítem 10 (Habla excesiva para mantener la cercanía) & .507 & -.200 \\
\hline Ítem 11 (Preocupación por otros cuando están ausentes) & .516 & -.125 \\
\hline Ítem 12 (Dificultades de sueño en soledad) & .555 & .494 \\
\hline Ítem 13 (Mejoría sueño cuando personas cerca) & .495 & .283 \\
\hline Ítem 14 (Ansiedad por separación o lejanía de personas) & .684 & .101 \\
\hline Ítem 15 (Pesadillas por lejanía del hogar) & .664 & -.078 \\
\hline Ítem 16 (Preocupación por pensamiento catastrofistas) & .549 & .119 \\
\hline Ítem 17 (Molestias por cambios de rutina si afecta a relaciones) & .572 & -.225 \\
\hline Ítem 18 (Preocupación por abandono de personas cercanas) & .620 & -.061 \\
\hline Ítem 19 (Mejoría del sueño si hay luces encendidas) & .422 & .475 \\
\hline Ítem 20 (Evitación de soledad en casa) & .549 & .477 \\
\hline Ítem 21 (Ansiedad o ataque de ansiedad por lejanía de personas) & .739 & .017 \\
\hline Ítem 22 (Ansiedad por no contacto telefónico) & .652 & .057 \\
\hline Ítem 23 (Temor por abandono de alguien cercano) & .569 & .003 \\
\hline Ítem 24 (Ocurrencia de ataques de pánico ante soledad) & .716 & -.004 \\
\hline Ítem 25 (Preocupación excesiva por pensamientos de daño ajeno) & .642 & -.028 \\
\hline Ítem 26 (Comentarios negativos por habla excesiva) & .341 & -.070 \\
\hline Ítem 27 (Preocupación por problemas ajenos a causa propia) & .484 & -.211 \\
\hline Valor propio & 8.668 & 1.303 \\
\hline Varianza explicada (\%) & 32.103 & 4.825 \\
\hline
\end{tabular}

Se replicó el análisis de ejes principales sin restricción de número de factores. El criterio de inclusión de los ítems, de acuerdo con lo establecido por los autores, fue una carga factorial mínima de .35. La solución mostró una estructura de seis factores, donde el primer factor explicaba el $34 \%$ de la varianza y sobre este cargaban todos los ítems en un rango de .36 a .75 (cargando el total de los ítems entre .5 y .75). El resto de los factores explicaban menos del $7 \%$ de la varianza cada uno de ellos. La rotación ortogonal que se aplicó a la solución de los seis factores no permitió mejorar la estructura factorial mejorando las cargas en los factores adicionales. Estos resultados, son muy similares a los alcanzados en el artículo original, por lo que parece ser que la escala se comportó de manera análoga tanto en la muestra española como en la anglosajona.

\section{Pulido de ítems para el desarrollo de una versión abreviada de la escala de 27 ítems}

Los resultados del análisis factorial se muestran en la Tabla 2, la cual muestra las cargas factoriales de cada ítem en los dos factores que obtuvieron valores propios superiores a 1 .
Podemos observar que solo 10 ítems cargan por encima de .60 en el primer factor. Aparece un segundo factor con un valor propio de 1.303. Se observan cargas cruzadas entre ambos factores en algunos ítems (particularmente, en ítems 7, 8 10, $12,13,17,19,20$ y 27). El primer factor explicaría el $32.1 \%$ de la varianza; el segundo, explicaría el 4.83 de la varianza. El resto de los ítems tanto del primer factor como del segundo mostraron cargas inferiores a este umbral. Sin embargo, el gráfico de sedimentación evidenció la idoneidad de considerar una estructura unifactorial en la retención de factores (Figura 1).

Cuando se aplicó el análisis factorial de ejes principales con los ítems seleccionados de la escala original tras el primer análisis factorial, el ajuste de la estructura fue considerablemente mejor, explicando el $45.3 \%$ de la varianza en una estructura unifactorial (Figura 2). El valor KMO de la nueva escala abreviada fue de $.919(p<.001)$

La consistencia interna de la escala ASA de 10 ítems fue elevada, $\alpha=.89$ (95\%IC [.87, .90]), y no aumentaba si se excluía ninguno de los ítems (ver Tabla 3). 


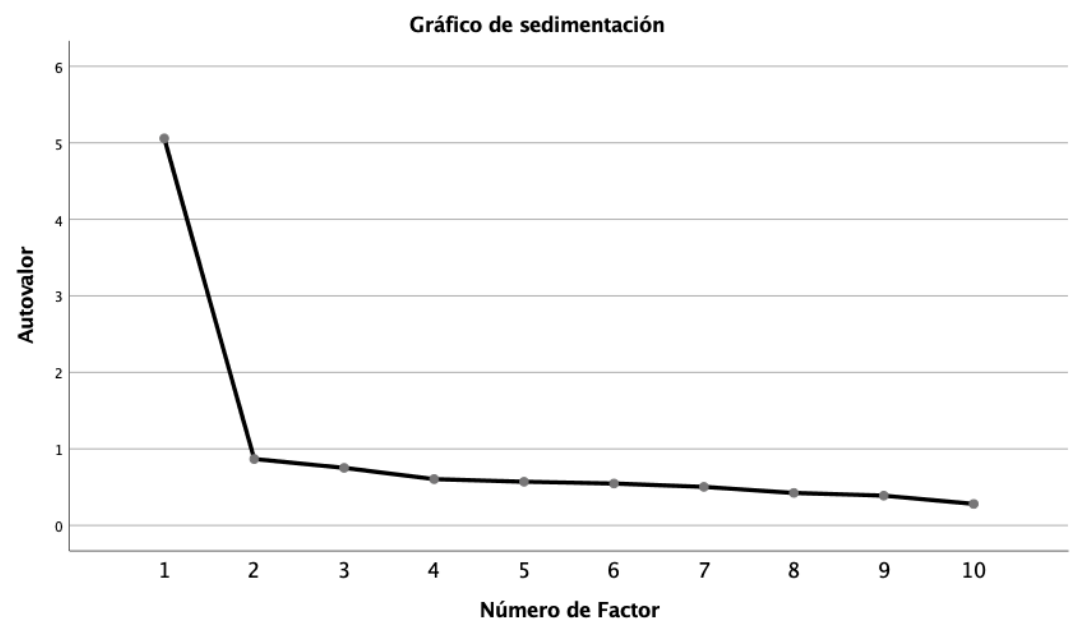

Figura 2. Gráfico de sedimentación del análisis factorial exploratorio por ejes principales de la versión reducida de diez ítems del ASA (ASA-10)

Tabla 3. Coeficiente $\alpha$ de Cronbach de la escala ASA abreviada si se excluye el ítem

\begin{tabular}{lc}
\hline & $\alpha$ de Cronbach \\
\hline ASA-10 & .886 \\
Ítems & .881 \\
$\quad$ Estrés ante un viaje largo & .876 \\
$\quad$ Sueño y pesadillas por separación & .871 \\
$\quad$ Estrés ante viaje de persona cercana & .873 \\
Ansiedad por separación o lejanía de & .876 \\
personas & .882 \\
Pesadillas por lejanía del hogar & \\
Preocupación por abandono de & .869 \\
personas cercanas & .875 \\
Ansiedad o ataque de ansiedad por & .871 \\
lejanía de personas & \\
Ansiedad por no contacto telefónico & \\
Ocurrencia de ataques de pánico ante & .876 \\
soledad & Preocupación excesiva por \\
pensamientos de daño ajeno & \\
\hline
\end{tabular}

\section{Correlación entre las versiones ASA-27, ASA-} 10 y otras medidas relacionadas

Como en la escala original, la puntuación total de la escala abreviada se obtuvo a partir del sumatorio de los ítems. Con el fin de descartar una función moduladora, se exploró si existía una correlación entre esta puntuación y la edad del sujeto. La correlación entre la edad y la puntuación total de la escala abreviada fue no significativa $(r=.08, p=.115)$. De igual modo, se calculó la correlación entre la puntuación total del ASA-27 y la del ASA-10, obteniendo una correlación positiva muy elevada $(r=.94, p<.001)$. A continuación, se llevaron a cabo análisis de correlación entre las puntuaciones totales de la escala ASA en sus dos formatos, y los índices obtenidos de las medidas incluidas en la batería de pruebas (ver Tabla 4). Se puede observar que el patrón de correlaciones entre las versiones de ASA y el resto de las medidas empleadas son similares. Resulta singular que la correlación entre la dimensión del STAI en ansiedad estado y la puntuación total en ASA-10 sí haya mostrado ser significativa, mientras que no lo haya hecho entre el constructo y el ASA-27. En suma, no hay pérdida de propiedades psicométricas a nivel de validez convergente si se aplica la versión reducida del ASA.

Tabla 4. Coeficientes de correlación entre las versiones de ASA (original y reducida) y cuestionarios y subescalas relacionadas

\begin{tabular}{|c|c|c|}
\hline & ASA-27 & ASA-10 \\
\hline \multicolumn{3}{|l|}{ STAI } \\
\hline Estado & .04 & $.35^{* *}$ \\
\hline Rasgo & $.52 * *$ & $.53 * *$ \\
\hline \multicolumn{3}{|l|}{ CAA } \\
\hline Baja autoestima & $.44 * *$ & $.37 * *$ \\
\hline Resolución hostil & $.37 * *$ & $.28 * *$ \\
\hline Expresión sentimientos & -.05 & -.04 \\
\hline $\begin{array}{l}\text { Autosuficiencia } \\
\text { emocional }\end{array}$ & $.18 * *$ & $.11^{*}$ \\
\hline \multicolumn{3}{|l|}{$\mathrm{CDE}$} \\
\hline Ansiedad separación & $.65^{* *}$ & $.63 * *$ \\
\hline $\begin{array}{l}\text { Expresión afectiva } \\
\text { pareja }\end{array}$ & $.55 * *$ & $.50 * *$ \\
\hline Modificación planes & $.42 * *$ & $.40 * *$ \\
\hline Miedo soledad & $.59 * *$ & $.57 * *$ \\
\hline Expresión límite & $.54 * *$ & $.53 * *$ \\
\hline Búsqueda atención & $.54 * *$ & $.51 * *$ \\
\hline
\end{tabular}

Nota. ASA-27=Cuestionario de Ansiedad por Separación en el Adulto; ASA-10=Cuestionario de Ansiedad por Separación de Adulto versión reducida (10 ítems). STAI=Inventario de Ansiedad Estado-Rasgo; CAA=Cuestionario de Apego Adulto (CAA); CDS=Cuestionario de Dependencia Emocional (CDE). $* p<.05 ; * * p<.01$ 
Diferencia de puntuación media de la escala ASA-10 por segmentación sociodemográfica.

Se calculó el estadístico $U$ de Mann-Whitney para comparativa entre dos grupos y el estadístico $H$ de Kruskal-Wallis para comparativa multigrupo. El tamaño del efecto se calculó con la correlación biserial de rangos. No se observaron diferencias en la puntuación total en la escala ASA-10 por sexo $(p=.431)$, estado civil $(p=.465)$, ocupación laboral $(p=.142)$, nivel de estudios $(p=.366)$, ni residencia $(p=.706)$.

\section{Discusión}

El objetivo principal de este estudio fue la adaptación de la escala ASA-27 a una versión más reducida de esta, sin que se vieran afectadas significativamente las propiedades psicométricas de la nueva escala. Como se ha podido determinar a través del presente estudio, la escala abreviada ASA-10, constituida por diez ítems pulidos a partir de la escala ASA-27, muestra una estructura factorial equivalente tanto a la versión original como a la versión adaptada a población turca (Dirioz et al., 2011), portuguesa (Ruiz-García et al., 2021) y española (Ruiz-García et al., 2019) (a partir, además, de criterios de inclusión más estrictos), caracterizada por una estructura unifactorial que permite el cálculo de una puntuación total (Manicavasagar et al., 2003; Ruiz-García et al., 2019, 2021). Estos resultados apoyan la evidencia empírica de que los síntomas propios del TASA configuran un constructo coherente. Una vez seleccionados los diez ítems de la escala ASA-10, el análisis factorial mostró que un factor único explicaba el $45.3 \%$ de la varianza, lo que era muy superior al $34.0 \%$ de la varianza que explicaba la estructura factorial de la escala ASA-27 en su adaptación a población española, y similar al $45.0 \%$ de la escala ASA-27 original. Estos resultados aportan una evidencia sólida a la estructura unifactorial de la escala abreviada, que superaría incluso la adaptada al español.

Los análisis de validez convergente arrojaron resultados similares entre las dos versiones de la escala ASA adaptada a población española, con correlaciones significativas que fueron de moderadas a altas. Además, la correlación entre ambos modelos de ASA fue muy elevada, alcanzando el coeficiente de correlación de .94. En cuanto a la validez convergente, cabe resaltar la diferencia encontrada en este estudio frente al del ASA-27 en español (Ruiz-García et al., 2019). Así el ASA-10 ha presentado correlaciones con el cuestionario STAI en su subescala estado, algo que no ocurría en el estudio anterior pero que sí se observó en el ASA-27 validado en población portuguesa con la edad ajustada (Ruiz-García et al., 2021).

Sin embargo, este estudio tiene algunas limitaciones que deberían ser exploradas en futuras investigaciones. Entre ellas cabe destacar que de los instrumentos empleados en la validación, el $\mathrm{CDE}$, se ha empleado en su construcción original validada en población colombiana, no existiendo estos datos para población española. $\mathrm{Y}$, en cuanto al STAI estado/rasgo, se ha observado en un estudio reciente (Guillén-Riquelme \& Buela-Casal, 2015) que es discutible la factorización de la escala como estado y como rasgo. De hecho, se han encontrado problemas en la validez de contenido, ya que aparecen otros posibles factores que además estarían fuertemente correlacionados con depresión evaluada mediante el cuestionario de depresión de Beck. Otra de las limitaciones es que, a pesar de presentar una muestra amplia, no ha resultado ser lo suficientemente heterogénea como para poder obtener valores normativos representativos de la población española. Además, sería necesario llevar a cabo análisis factoriales confirmatorios que puedan validar la estructura factorial de la escala, así como hacer una posible comparativa de las bondades de ajustes de la misma escala con estructuras factoriales alternativas. En futuros estudios, sería también aconsejable contrastar otras posibles estructuras de la escala a través de procesos de selección ítems bajo criterios más asociados a la validez de contenido, a diferencia del presente estudio, que ha empleado criterios estadísticos para dicho proceso de pulido de ítems. Por último, futuros estudios deberán llevar a cabo análisis de sensibilidad y análisis discriminativos detallados que permitan contrastar su eficacia en la detección de perfiles de riesgo de presentar cuadros clínicamente significativos de ansiedad por separación en adultos, tanto sobre población general, como sobre poblaciones clínicas. 
Este estudio es un primer paso en el extenso proceso de adaptación y validación de escalas abreviadas con fines clínicos, que deberán llevar a cabo futuras investigaciones en diferentes poblaciones y con muestras más amplias. En el caso de la ansiedad por separación en adultos este proceso sería interesante realizarse en contexto europeo, ya que los estudios apuntan a una mayor prevalencia en estos (Silove et al., 2015).

Finalmente, la escala ASA-10 ha mostrado unas buenas propiedades psicométricas que sugieren que es una escala sólida metodológicamente, potencialmente útil como escala de cribado clínico, pudiendo ser una herramienta rigurosa como primera línea en procesos de evaluación psicopatológica general. La brevedad de la escala, producto del proceso de pulido y acortamiento, no ha ido en detrimento de la calidad de sus propiedades psicométricas.

\section{Referencias}

American Psychiatric Association (APA) (2014). Manual diagnóstico y estadístico de los trastornos mentales (DSM-5), $5^{\mathrm{a}}$ Ed. Madrid: Editorial Médica Panamericana.

Baldwin, D. S., Gordon, R., Abelli, M., \& Pini, S. (2016). The separation of adult separation anxiety disorder. CNS Spectrums, 21, 289294. doi: $10.1017 / \mathrm{S} 1092852916000080$

Buela-Casal, G., Guillén-Riquelme, A., \& Seisdedos-Cubero, N. (2015). Stai. Cuestionario de Ansiedad Estado-Rasgo. TEA Ediciones.

Cohen, J., Cohen, P., West, S. G., \& Aiken, L. S. (2002). Applied multiple regression / correlation analysis for the behavioral sciences $\left(3^{\mathrm{a}}\right.$ ed.). London, England: Routledge.

Devici, H. (2019). Parental Acceptance-Rejection and Adult Separation Anxiety: The Mediation of Adult Attachment Insecurity. SAGE Open, 9(4) 1-9. doi:10.1177/2158244019885138

Dirioz, M., Alkin, T., Yemez, B., Onur, E., \& Eminagaoglu, B. (2011). The validity and reliability of Turkish version of Separation Anxiety Symptom Inventory and Adult Separation Anxiety Questionnaire. Turkish
Journal of Psychiatry, 23(2), 108-116. doi:10.5080/u6364

Dowsett, E., Delfabbro, P., \& Chur-Hansen, A. (2020). Adult separation anxiety disorder: The human-animal bond. Journal of Affective Disorders, 270, 90-96. doi:10.1016/j.jad.2020.03.147

Fabrigar, L. R., Wegener, D. T., MacCallum, R. C., \& Strahan, E. J. (1999). Evaluating the use of exploratory factor analysis in psychological research. Psychological Methods, 4(3), 272299. doi:10.1037/1082-989X.4.3.272

Fernández-Marcos, T. (2014). Un caso de dolor de estómago y ansiedad por separación en una estudiante universitaria: La relevancia de la funcionalidad en la explicación del problema. XII Encuentro de Servicios Psicológicos y Psicopedagógicos Universitarios (SPPU), España, Valencia.

Gesi, C., Abelli, M., Cardini, A., Lari, L., Di Paolo, L., Silove, D., \& Pini, S. (2015). Separation anxiety disorder from the perspective of DSM-5: Clinical investigation among subjects with panic disorder and associations with mood disorders spectrum. CNS Spectrums, 21(1), 70-75. doi:10.1017/S1092852914000807

Guillén-Riquelme, A., \& Buela-Casal, G. (2015). Estructura factorial del Cuestionario de Ansiedad Estado-Riesgo (STAI) para pacientes diagnosticados con depresión. Salud Mental, 38(4), 293-298. https://doi.org/10.17711/sm.01853325.2015.040

Greer, F., \& Liu, J. (2016). Creating short forms and screening measures. In K. Schweizer and C. DiStefano (Eds.) Principles and methods of test construction: Standards and recent advances, vol 3. Hogrefe Publishing.

Hale, W. W., Berg, A., \& Malhotra, S. (2018). An examination of separation anxiety disorder symptoms in adults from a clinical population. Journal of Systems and Integrative Neuroscience, 4(2), 1-5. doi:10.15761/JSIN.1000198

JASP Team (2019). JASP (Version 0.11.1) [Computer software].

Kemper, C. J., Trapp, S., Kathmann, N., Samuel, D. B., \& Ziegler, M. (2019). Short versus long scales in clinical assessment: Exploring the 
trade-off between resources saved and psychometric quality lost using two measures of obsessive-compulsive symptoms. Assessment, 26(5), 767-782.

Kruyen, P. M., Emons, W. H. M., \& Sijtsma, K. (2013). On the shortcomings of shortened tests: A literature review. International Journal of Testing, 13(3), 223-248. doi:10.1080/15305058.2012.703734

Lemos-Hoyos, M., \& Londoño-Arrendondo, N. H. (2006). Construcción y validación del cuestionario de dependencia emocional en población colombiana. Acta Colombiana de Psicología, 9(2), 127-140.

Mabilia, D., Di Riso, D., Liso, A., \& Bobbio, A. (2019). A prediction model for separation anxiety: The role of attachment styles and internalizing symptoms in Italian young adults. Journal of Adult Development, 26(4), 286-294. doi:10.1007/s10804-019-09327-y

Manicavasagar, V., Silove, D., Wagner, R., \& Drobney, J. (2012). Adult Separation Anxiety Questionnaire - ASA-27. Measurement instrument database for the social science. Recuperado de www.midss.ie. dx.doi.org/10.13072/midss.162

Manicavasagar, V., Silove, D., \& Curtis, J. (1997). Separation anxiety in adulthood: A phenomenological investigation. Comprehensive Psychiatry, 38(5), 274-282. doi:10.1016/S0010-440X(97)90060-2

Manicavasagar, V., Silove, D. Wagner, R., \& Drobney, J. (2003). A self-report questionnaire for measuring separation anxiety in adulthood. Comprehensive Psychiatry, 44(2), 146-153. doi.org/10.1053/comp.2003.50024

Manicavasagar, V., Silove, D., \& Curtis, J. (1997). Separation anxiety in adulthood: A phenomenological investigation. Comprehensive Psychiatry, 38(5), 274-282. doi:10.1016/S0010-440X(97)90060-2

Manicavasagar, V., \& Silove, D. (2020a). Making a diagnosis of separation anxiety disorder in adulthood. In: Separation anxiety disorders in adults: Clinical features, diagnostic dilemmas and treatment guidelines (pp. 19-29). Academic Press - Elsevier. doi:10.1016/C2016-0-01828-4.
Manicavasagar, V., \& Silove, D. (2020b). Comorbidity with other disorders and conditions. In: Separation Anxiety Disorders in Adults: Clinical Features, Diagnostic Dilemmas and Treatment Guidelines (pp. 151170). Academic Press - Elsevier. doi:10.1016/C2016-0-01828-4.

McDonald, R. P. (1999). Test theory: A unified treatment. Erlbaum.

Melero, R., \& Cantero, M. J. (2008). Los estilos afectivos en la población española: Un cuestionario de evaluación del apego adulto. Clínica y Salud, 19(1), 83-100.

Posavac, S. S., \& Posavac, H.D. (2017). Adult separation anxiety disorder symptomology and susceptibility to marketing persuasion. Journal of Social and Clinical Psychology, 36(2), 158-169.

doi:10.1521/jscp.2017.36.2.158

Pozzi, G., Bruschi, A., De Angelis, A., Pascucci, M., Hatzigiakoumis, D.S., Grandinetti, P., ... \& Janiri, L. (2014). Adult separation anxiety and TCI-R personality dimensions in patients with anxiety, alcohol use, and gambling: A preliminary report. Biomed Research International, 680985. doi:10.1155/2014/680985

Pozzi, G., Bruschi, A., De Angelis, A., Pascucci, M., Hatzigiakoumis, D. S., Grandinetti, P., ... \& Janiri, L. (2014). Adult separation anxiety and TCI-R personality dimensions in patients with anxiety, alcohol use, and gambling: A preliminary report. Biomed Research International, 680985. doi:10.1155/ 2014/680985

Román, M., Hodges, J., Palacios, J., Moreno, C., \& Hillman, S. (2015). Evaluación de las representaciones mentales de apego a través de historias incompletas: Aplicación española de Story Stem Assessment Profile (SSAP). Revista Iberoamericana de Diagnóstico y Evaluación - e Avaliação Psicológica, 46(1), 5-19. doi:10.21865/RIDEP46.1.01

Ruiz, A., \& Rando, M. A. (2015). Tratamiento de un caso de ansiedad por separación en una mujer adulta. XIII Encuentro de Servicios Psicológicos $\quad y \quad$ Psicopedagógicos Universitarios (SPPU), España, Madrid.

Ruiz-García, A., Jiménez, Ó., Fenollar-Cortés, J., Cano-Marín, J., Ojeda-Mora, M. A., ... \& 
Valero-Aguayo, L. (2019). Adaptación y validación al español del Cuestionario de Ansiedad por Separación en el Adulto (ASA27). Revista Iberoamericana de Diagnóstico y Evaluación Psicológica - e Avaliação Psicológica, 53(4), 153-167. doi:10.21865/RIDEP53.3.12

Ruiz-García, A., Jiménez, Ó., Resurrección, D.M., Ferreira, M., Reis-Jorge, J., \& FenollarCortés, J. (2021). Portuguese validation of the Adult Separation Anxiety - Questionnaire (ASA-27). PLoS ONE, 16(3), e0248149.

doi:10.1371/journal.pone.0248149

Schneier, F. R., Moskow, D. M., Choo, T-H., Galfalvy, H., Campeas, R., \& Sanchez-Lacay, A. (2017). A randomized controlled pilot trial of vilazodone for adult separation anxiety disorder. Depression and Anxiety, 34(12), 1085-1095 doi:10.1002/da.22693

Schweizer, K., \& DiStefano, C. (Eds.). (2016). Principles and methods of test construction: Standards and recent advances (Vol. 3). Hogrefe Publishing

Silove, D., Alonso, J., Bromet, E., Gruber, M., Sampson, N., Scott, K., ... \& Kessler, R.C. (2015). Pediatric-onset separation and Adultonset Separation anxiety disorder across countries in the World Mental Health Survey. The American Journal of Psychiatry, 172, 647-656. doi:10.1176/appi.ajp.2015.14091185

Spielberger, C. D., Gorsuch, R. L., \& Lushene, R. E (1970). Manual for the State-Trait Anxiety Inventory (Self-evaluation questionnaire). Palo Alto, CA: Consulting Psychologists Press.

Streiner, D. L. (2003). Starting at the beginning: An introduction to coefficient alpha and internal consistency. Journal of personality assessment, 80(1), 99-103.

Tavakol, M., \& Dennick, R. (2011). Making sense of Cronbach's alpha. International Journal of Medical Education, 2, 53-55. 